\title{
Medium-term outcome after anomalous aortic origin of a coronary artery repair in a pediatric cohort
}

\author{
Carol A. Wittlieb-Weber, MD, ${ }^{\text {a }}$ Stephen M. Paridon, MD, ${ }^{a}$ J. William Gaynor, MD, \\ Thomas L. Spray, MD, ${ }^{b}$ David R. Weber, MD, ${ }^{c}$ and Julie A. Brothers, MD ${ }^{a}$
}

\begin{abstract}
Background: Anomalous aortic origin of a coronary artery with an interarterial and intramural course (AAOCA) is a rare anomaly with increased risk of sudden cardiac death during or just after exercise among otherwise healthy youth. Risk stratification and management remain controversial, especially for the asymptomatic child with an anomalous right coronary artery from the left coronary sinus (ARCA). Medium-term surgical and quality-of-life (QOL) outcome data are lacking in this population.
\end{abstract}

\begin{abstract}
Methods: We performed medical record review on 24 subjects who underwent AAOCA repair between 2001 and 2007 at The Children's Hospital of Philadelphia. QOL was prospectively assessed with age-appropriate questionnaires.

Results: Median age at follow-up was 18 (range, 11-25) years, median follow-up from surgery was 63 (range, 12-110) months, and $16(67 \%)$ had ARCA. All were alive without exercise restriction. Thirteen (54\%) complained of cardiac-type symptoms postoperatively, most commonly chest pain, none correlating with evidence of ischemia on testing. Of the 13 patients, 7 (54\%) reported the same symptoms preoperatively; and of these, 5 had ARCA. Postoperative morbidity occurred in $16(67 \%)$, including pericardial effusion $(\mathrm{n}=11)$, wound infection $(\mathrm{n}=2)$, and development of mild aortic insufficiency $(\mathrm{n}=4)$. QOL questionnaires were sent to 21 subjects; 12 (57\%) were returned. Average QOL was normal for all subjects.

Conclusions: In the medium-term after AAOCA repair, cardiac-type symptoms frequently persist and morbidity is common, but these do not impair QOL. The significance of these findings in the long-term is unknown and warrants continued follow-up. (J Thorac Cardiovasc Surg 2014;147:1580-6)
\end{abstract}

Anomalous origin of a coronary artery from the opposite sinus of Valsalva with an interarterial and intramural course (AAOCA) is a rare coronary artery variation associated with myocardial ischemia and sudden cardiac death (SCD) in the young during or just after exercise. ${ }^{1}$ The incidence of AAOCA varies, ranging from $0.17 \%$ in autopsy series to $1.2 \%$ in patients angiographically evaluated. ${ }^{2-6}$ AAOCA is the second most common cause of SCD in young athletes, ${ }^{7}$ yet the exact mechanism of ischemia contributing to the risk of SCD and the true incidence of SCD remain unknown. Ostial stenosis, an oblique takeoff of the anomalous coronary, and compression of the coronary between the great arteries are possible risk factors that can limit coronary reserve. In addition, the presence or absence of an intramural course has been considered a potential independent risk factor for myocardial ischemia. ${ }^{8,9}$

From the Divisions of Cardiology, ${ }^{\mathrm{a}}$ Cardiothoracic Surgery, ${ }^{\mathrm{b}}$ and Endocrinology, The Children's Hospital of Philadelphia, Philadelphia, Pa.

This study was funded by the Department of Cardiology at The Children's Hospital of Philadelphia.

Disclosures: Authors have nothing to disclose with regard to commercial support.

Received for publication Nov 19, 2012; revisions received June 14, 2013; accepted for publication July 2, 2013; available ahead of print Aug 28, 2013.

Address for reprints: Carol A. Wittlieb-Weber, MD, 34th and Civic Center Boulevard, Main Hospital, 8NW25, Philadelphia, PA 19104 (E-mail: wittliebc@email.chop. edu).

$0022-5223 / \$ 36.00$

Copyright (c) 2014 by The American Association for Thoracic Surgery http://dx.doi.org/10.1016/j.jtcvs.2013.07.022
Many patients with AAOCA have symptoms, such as palpitations and chest pain, that occur frequently in the young, even in the absence of coronary anomalies. ${ }^{10}$ When these symptoms occur with exertion, there is a higher index of suspicion for cardiac pathologic features, often prompting an echocardiogram. Significant controversy exists regarding treatment of asymptomatic patients, especially those with an anomalous right coronary artery from the left sinus of Valsalva with an interarterial, intramural course (ARCA), where the reported risk of SCD is lower compared with ALCA. ${ }^{8,11,12}$ Concern has been raised regarding persistent cardiac symptoms ${ }^{13}$ and abnormal findings on exercise stress testing and noninvasive imaging after surgical repair of AAOCA. ${ }^{14,15}$ Quality of life (QOL), however, has been normal in the short-term after AAOCA repair. ${ }^{15}$ There are limited medium-term surgical outcome data with AAOCA, and none focusing solely on the pediatric population with interarterial, intramural AAOCA. ${ }^{8}$ The purpose of this study was to evaluate medium-term postoperative outcomes and QOL in a small pediatric cohort after AAOCA repair.

\section{PATIENTS AND METHODS Subjects}

After approval from the Institutional Review Board at The Children's Hospital of Philadelphia (Philadelphia, Pa), subjects were identified from a cohort with surgically repaired AAOCA previously evaluated at our 


\section{Abbreviations and Acronyms \\ AAOCA $=$ anomalous aortic origin of a coronary artery with an interarterial and intramural course \\ ALCA = anomalous left coronary artery \\ ARCA = anomalous right coronary artery \\ $\mathrm{CHQ}=$ Child-Health Questionnaire \\ CF87 = Child-Completed 87-Item Form \\ ECG = electrocardiogram \\ EST $=$ exercise stress testing \\ MPS = myocardial perfusion scan \\ PedsQL $=$ Pediatric Quality of Life Inventory 4.0 \\ PF50 = Parent-Completed 50-Item Form \\ $\mathrm{QOL}=$ quality of life \\ SCD = sudden cardiac death \\ $\mathrm{SE} \quad=$ stress echocardiogram}

institution. ${ }^{14,15}$ Only subjects who were part of this cohort were approached for enrollment. All had AAOCA with an interarterial, intramural course, and underwent surgical repair at The Children's Hospital of Philadelphia between 2001 and 2007. A waiver of consent was obtained for the retrospective medical record review portion of this study. Informed consent was obtained over the telephone from each subject (or guardian if $<18$ years of age) for prospective QOL evaluation.

\section{Clinical Data}

Each subject's medical record was reviewed for presenting symptoms, operative details and postoperative complications, study results, and cardiac-type symptoms. Cardiac-type symptoms were defined as those potentially referable to the cardiovascular system (chest pain, palpitations, syncope, or lightheadedness with or without exertion) occurring 3 months or later after surgical repair and prompting medical evaluation. The decision to include resting symptoms was because several subjects presented initially with resting symptoms only $(\mathrm{n}=7)$.

\section{QOL Questionnaires}

Standardized questionnaires were sent to subjects who gave consent. Subjects younger than 18 years completed a Child-Health Questionnaire (CHQ) Child-Completed 87-Item Form (CHQ-CF87) and an age-appropriate Pediatric Quality of Life Inventory 4.0 (PedsQL) self-report. Parents of subjects younger than 18 years completed a CHQ Parent-Completed 50-Item Form (CHQ-PF50) and a PedsQL parent-proxy report. Subjects 18 years and older completed a PedsQL young-adult report and a QualityMetrics SF-36v2 survey. All scores were generated based on questionnaire protocol and compared with published normative values. ${ }^{16-19}$ A higher score signified better selfperceived function.

\section{Statistical Analysis}

Baseline clinical characteristics were described using standard descriptive statistics. Mean QOL questionnaire scores were compared with normal values from healthy children ${ }^{16-19}$ using a 2-sample, 2-tailed $t$ test with equal or unequal variances, as appropriate. $P<.05$ was considered statistically significant. All statistical analyses were conducted with Stata 12 (StataCorp, College Station, Tex).

\section{RESULTS \\ Baseline Demographics}

Twenty-five subjects met criteria for study inclusion. Twenty-four $(96 \%)$ were included in the retrospective medical record review; one subject's medical record could not be obtained. Subject characteristics are summarized in Table 1.

\section{Surgical Repair and Postoperative Complications}

Twenty-three subjects underwent the unroofing procedure, and 1 subject (ARCA) had coronary reimplantation due to a short intramural course believed not amenable to unroofing. In $16(70 \%)$ of subjects, the aortic commissure was detached and resuspended after unroofing. All survived the operation. One ALCA subject required emergent reoperation on the first postoperative night for an aortic incision disruption that was successfully repaired. Eleven $(46 \%)$ of subjects developed pericardial effusions postoperatively (ARCA, $\mathrm{n}=6$; ALCA, $\mathrm{n}=5$ ), one of whom (ALCA) experienced cardiac tamponade 16 days postoperatively, requiring operative drainage. Of the remaining 10 subjects with pericardial effusions, 5 were small, 4 were initially small but progressed to moderate, and 1 was moderate at diagnosis. Of the 10 subjects, 7 were treated with ibuprofen (Motrin), and all were monitored until the effusion resolved. Two ARCA subjects developed superficial wound infections postoperatively that were managed with oral antibiotics.

\section{Postoperative Management}

All subjects were alive at the most recent evaluation (median, 63 [range, 12-110] months after surgical repair), and none was restricted from exercise. Ten $(42 \%)$ of subjects participated in organized sports, with 2 competing in collegiate athletics. The frequency of recommended cardiac follow-up was variable, ranging from every 6 months to every 4 years, with a median of 1 year. Eight $(33 \%)$ of subjects were overdue for follow-up by longer than 1 year.

The type and frequency of postoperative testing varied. All subjects had an electrocardiogram (ECG) performed at their most recent cardiology evaluation, with $21(88 \%)$ interpreted as normal. Two subjects (ALCA, ARCA) had nonspecific T-wave changes and 1 (ARCA) had Q waves in leads V1, V2, aVR, and aVL. Twenty-two (92\%) of subjects underwent resting echocardiography at their most recent cardiology evaluation. All had normal systolic function. One ARCA subject developed mild hypokinesia of the anterior ventricular septum postoperatively; this same subject had nonspecific T-wave changes on the most recent ECG. Mild aortic insufficiency was a new finding in $5(21 \%)$ of subjects postoperatively; $4(80 \%)$ had ALCA, and 3 underwent aortic commissural resuspension 
TABLE 1. Subject characteristics $(n=24)$

\begin{tabular}{lc}
\hline \multicolumn{1}{c}{ Characteristic } & Value \\
\hline Age at surgery, y & $12(5-18)$ \\
Age at follow-up, y & $18(11-25)$ \\
Time to follow-up, mo & $63(12-110)$ \\
Male sex, \% & 71 \\
Coronary anatomy & ARCA, $16(67 \%) ;$ ALCA, 8 \\
Patient presentation & Symptomatic, $17 ;$ \\
& chest pain, $11^{*}:$ \\
& 7 exertion and 4 rest \\
& syncope/near syncope, $5^{*}$ : \\
& 3 exertion and 2 rest \\
& palpitations, 1 rest \\
& aborted cardiac arrest, 1 \\
& Asymptomatic, 7 \\
\hline
\end{tabular}

Data are given as median (range) unless otherwise indicated. $A R C A$, Anomalous right coronary artery; $A L C A$, anomalous left coronary artery. *One patient with chest pain and syncope.

during the operation. One of the 4 ALCA subjects who developed mild aortic insufficiency postoperatively had nonspecific T-wave changes on the most recent ECG. In addition, one ALCA subject with a bicuspid aortic valve developed mild aortic valve insufficiency postoperatively but did not have aortic commissural resuspension. All subjects had documentation of flow in the unroofed coronary artery, as shown on echocardiogram during the postoperative period.

Since our prospective evaluation of this cohort published in $2007,{ }^{14} 12(50 \%)$ of subjects have had additional exercise stress testing (EST). When the data from the 2 studies are combined, a total of $12(50 \%)$ of subjects have had abnormal findings. New findings since our last publication ${ }^{14}$ are shown in Table 2. The subject with ALCA who had mild basal anterior septal hypokinesis on stress echocardiogram (SE; 109 months after repair) was followed up at an outside institution and the decision to not pursue additional testing at the time was unknown. The subject with ARCA who had a small, fixed, anterior apical defect on myocardial perfusion scan (MPS; 29 months after repair) was followed up at our institution and lost to follow-up.

One ALCA subject, who was previously reported ${ }^{14}$ to have apical-septal and midanterior-septal hypokinesis on SE, 9 months after operative repair, underwent cardiac magnetic resonance imaging 57 months later, which showed no regional wall abnormalities. This patient had a normal MPS and no ECG changes with exercise at the time of his SE and was not referred for additional testing at the time. One ARCA subject, who was previously reported $^{14}$ to have a small, fixed, anterior septal defect on MPS, 3 months after operative repair, had 2 additional exercise stress tests (11 and 21 months after repair) that showed mild chronotropic impairment and mild paradoxical septal motion on MPS. Finally, 4 other subjects, all with ARCA, who had abnormal stress test results reported in our original cohort ${ }^{14}$ were lost to follow-up and, to our knowledge, repeat testing has not been performed. Abnormal findings in these subjects included fixed apical-inferior hypokinesis on SE (42 months after repair), anterolateral Q waves on EST (20 months after repair), and a blunted blood pressure response to exercise in 2 subjects ( 22 and 27 months after repair, respectively).

\section{Postoperative Symptoms}

Thirteen (54\%) of subjects complained of cardiac-type symptoms postoperatively, most commonly chest pain (Table 3). No symptoms correlated with evidence of

TABLE 2. Cardiac testing results

\begin{tabular}{|c|c|c|c|c|c|c|}
\hline ID & $\begin{array}{l}\text { Coronary } \\
\text { anatomy }\end{array}$ & $\begin{array}{l}\text { Presenting } \\
\text { symptoms } \\
\end{array}$ & $\begin{array}{c}\text { Time from } \\
\text { surgery, mo }\end{array}$ & Exercise test & Stress echo & $\begin{array}{c}\text { Myocardial } \\
\text { perfusion scan }\end{array}$ \\
\hline 5 & ALCA & None & 109 & Normal & $\begin{array}{c}\text { Mild basal anterior septal } \\
\text { hypokinesis }\end{array}$ & - \\
\hline $10 \mathrm{a}$ & ALCA & Syncope, exertion & 15 & Mild chronotropic impairment & Normal & Normal \\
\hline $10 \mathrm{~b}$ & & & 76 & Mild chronotropic impairment & Normal & - \\
\hline $11 \mathrm{a}$ & ARCA & None & 15 & Normal & Normal & Small inferoseptal defect \\
\hline $11 b$ & & & 39 & Normal & - & Normal \\
\hline $11 \mathrm{c}$ & & & 80 & Normal & - & Normal \\
\hline $23 \mathrm{a}$ & ARCA & Chest pain, exertion & 4 & Normal & - & $\begin{array}{c}\text { Mild reversible anterior wall } \\
\text { defect }\end{array}$ \\
\hline $23 b$ & & & 8 & Normal & - & Normal \\
\hline $22 \mathrm{a}$ & ARCA & Syncope, exertion & 3 & Normal & Normal & $\begin{array}{l}\text { Small, fixed, anterior septal } \\
\text { defect, paradoxical septal } \\
\text { motion }\end{array}$ \\
\hline $22 b$ & & & 11 & Mild chronotropic impairment & - & Mild paradoxical septal motion \\
\hline $22 \mathrm{c}$ & & & 21 & Mild chronotropic impairment & - & Mild paradoxical septal motion \\
\hline $24 \mathrm{a}$ & ARCA & Chest pain, exertion & 3 & Normal & Normal & Normal \\
\hline $24 b$ & & & 29 & Normal & Normal & Small, fixed anterior apical defect \\
\hline
\end{tabular}

$I D$, Identification; $A L C A$, anomalous left coronary artery; ARCA, anomalous right coronary artery. 
TABLE 3. Postoperative symptoms and symptom evaluation

\begin{tabular}{|c|c|c|c|c|c|c|}
\hline ID & $\begin{array}{r}\text { Coronary } \\
\text { anatomy } \\
\end{array}$ & $\begin{array}{l}\text { Presenting } \\
\text { symptoms }\end{array}$ & $\begin{array}{l}\text { Postoperative symptoms } \\
\text { (time from surgery, mo) }\end{array}$ & $\begin{array}{c}\text { Symptom } \\
\text { evaluation* }\end{array}$ & $\begin{array}{l}\text { Significant } \\
\text { findings }\end{array}$ & $\begin{array}{l}\text { Symptoms at most recent } \\
\text { visit (time from surgery, mo) }\end{array}$ \\
\hline $1 \mathrm{a}$ & ARCA & Chest pain, exercise & Chest pain (48) & EST, SE, MPS & - & None (106) \\
\hline $1 \mathrm{~b}$ & & & Chest pain, rest (83) & ED evaluation $\dagger$ & & \\
\hline $1 \mathrm{c}$ & & & Syncope, rest (91) & ED evaluation $\dagger$ & & \\
\hline $3 a$ & ARCA & Presyncope, rest & Presyncope, rest (5) & ED evaluation: ECG & - & Syncope, rest (61) \\
\hline $3 b$ & & & Chest pain, rest (13) & None & & \\
\hline $3 c$ & & & Syncope, rest (61) & Holter SE $\ddagger$ & - & \\
\hline 5 & ALCA & None & Chest pain, rest (97) & None & & None (109) \\
\hline 7 & ALCA & Chest pain, rest & Chest pain, rest (40) & ED evaluation $\dagger$ & & None (48) \\
\hline 8 & ARCA & Chest pain, rest & Chest pain, rest (57) & None & & Chest pain, rest (57) \\
\hline 11 & ARCA & None & Chest pain, rest (59) & ED evaluation $\dagger$ & & None $(80)$ \\
\hline $13 \mathrm{a}$ & ARCA & None & Chest pain, exertion (17) & EST, SE & - & Lightheaded, exertion (85) \\
\hline $13 b$ & & & Palpitations (22) & Holter & - & \\
\hline $13 \mathrm{c}$ & & & Lightheaded, exertion (85) & None & & \\
\hline 15 & ARCA & Chest pain, exercise & Chest pain, exercise (14) & ED evaluation $\dagger$ & & None (64) \\
\hline 17 & ALCA & None & Chest pain (37) & ED evaluation $\dagger$ & & None (62) \\
\hline \multirow[t]{4}{*}{18} & ALCA & Aborted sudden death & Chest pain, rest (66) $\S$ & ECG & ST elevation & None (66) \\
\hline & & & & CRP & Elevated & \\
\hline & & & & TTE, MRI, & - & \\
\hline & & & & Troponin & - & \\
\hline $20 \mathrm{a}$ & ALCA & Chest pain, rest & Chest pain, rest (6) & Neurologic referral & - & None (48) \\
\hline $20 \mathrm{~b}$ & & & Chest pain, rest (26) & EST, MPS & - & \\
\hline $21 \mathrm{a}$ & ARCA & Syncope, rest & Syncope, rest (4) & $\begin{array}{l}\text { ED evaluation: ECG, CXR, } \\
\text { Echo }\end{array}$ & Small PE & None (67) \\
\hline $21 b$ & & & Syncope, rest (21) & ED evaluation $\dagger$ & & \\
\hline $21 \mathrm{c}$ & & & Syncope, rest (52) & Neurologic referral & - & \\
\hline 25 & ARCA & None & Chest pain, rest (4) & None & & None (40) \\
\hline
\end{tabular}

$I D$, Identification; $A R C A$, anomalous right coronary artery; $E S T$, exercise stress testing; $S E$, stress echocardiogram; $M P S$, myocardial perfusion scan; $E D$, emergency department; $E C G$, electrocardiogram; $C R P, \mathrm{C}$-reactive protein; TTE, transthoracic echocardiogram; $M R I$, magnetic resonance imaging; $C X R$, chest x-ray; $P E$, pericardial effusion; $A L C A$, anomalous left coronary artery. *Other than routine testing at cardiology visit. †Evaluated at outside ED (records unavailable). $\ddagger$ Not done, patient lost to follow-up. $\S$ Hospital admission for presumed pericarditis.

ischemia on testing, and no subject was restricted from exercise because of symptoms. Of the 13 subjects, 7 (54\%) reported the same symptoms preoperatively; and of these, 5 had ARCA. Five subjects with postoperative symptoms were asymptomatic before surgery. The actuarial survival and freedom from cardiac-type symptom curves for the 24 subjects are shown in Figure 1.

Of the 9 subjects who presented with exercise-induced symptoms (chest pain, $\mathrm{n}=6$; syncope, $\mathrm{n}=2$; chest pain and syncope, $\mathrm{n}=1), 2$ complained of chest pain with exercise postoperatively (6 weeks and 14 months after repair, respectively). One of these 2 subjects, who had a history of ARCA, complained of activity-induced chest pain 6 weeks after surgical repair, prompting referral for EST, which showed inferior lead ST depression (up to 5 $\mathrm{mm}$ ) during exercise, with no reported symptoms. Because of the ST abnormality, an MPS was performed, which was normal. He had repeat testing 16 months later, consisting of EST, SE, and MPS, all of which were normal. For this study, cardiac-type symptoms were defined as occurring 3 months or longer after surgical repair; therefore, this patient's data were not included in other analyses.

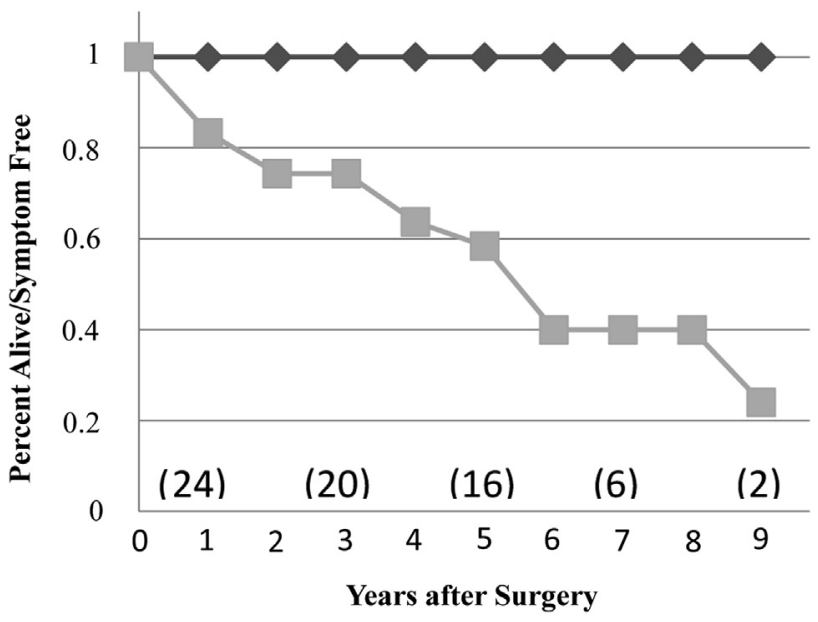

FIGURE 1. Freedom from death and cardiac-type symptoms. Actuarial curves demonstrating survival and freedom from cardiac-type symptoms for 24 patients who underwent surgical repair of anomalous aortic origin of a coronary artery with an interarterial and intramural course. Follow-up, in years, since operative repair is shown along the $x$ axis, and the number of patients available for follow-up is designated in parenthesis. 
TABLE 4. PedsQL results

\begin{tabular}{|c|c|c|c|c|c|c|c|}
\hline \multirow{2}{*}{$\frac{\text { Subjects }<18 \text { y }(n=8)}{\text { Health variable }}$} & \multicolumn{7}{|c|}{ Subjects $\geq 18$ y $(n=4)$} \\
\hline & Score $( \pm$ SD) & Normal value $( \pm$ SD) & $P$ value & Health variable & Score $( \pm$ SD) & Normal value $( \pm$ SD) & $P$ value \\
\hline Subject & & & & Subject & & & \\
\hline Physical & $92.0(9.3)$ & $85.2(13.7)$ & .16 & Physical & $96.9(4.0)$ & $86.3(10.6)$ & .046 \\
\hline Psychosocial & $92.5(10.3)$ & $78.2(14.9)$ & .007 & Psychosocial & $96.9(3.6)$ & $73.9(10.5)$ & $<.001$ \\
\hline Total & $92.4(9.1)$ & $80.6(13.6)$ & 01 & Total & $95.7(4.0)$ & $78.2(9.2)$ & $<.001$ \\
\hline \multicolumn{8}{|l|}{ Parent-proxy } \\
\hline Physical & 9.3 (12.9) & $78.5(22.3)$ & .13 & & & & \\
\hline Psychosocial & $89.7(9.0)$ & $76.1(16.3)$ & .02 & & & & \\
\hline Total & $89.6(8.7)$ & $76.9(16.8)$ & .03 & & & & \\
\hline
\end{tabular}

\section{QOL Questionnaires}

Twenty-one subjects consented to QOL questionnaires; $12(57 \%)$ were returned. On average, subjects scored in the normal range for all parameters evaluated, as shown in Table 4-7. There were no significant differences in age, sex, or coronary anatomy (ARCA versus ALCA) between those who returned completed QOL questionnaires and those who did not.

Subjects younger than 18 years had significantly higher psychosocial and total scores on the PedsQL than healthy controls for both the self-report and the parent-proxy report. On the CHQ-CF87, younger subjects scored significantly higher than healthy controls regarding physical functioning, behavior, social limits-behavior, and social limits-physical. Younger subjects appeared to have a mean score lower than the norm on the CHQ-CF87 for bodily pain. Two subjects, whose scores were low (20 and 40 of 100), experienced noncardiac pain. When these scores were excluded, the mean score for bodily pain increased to $81.7 \pm 11.7$. Although this value is higher than the mean for healthy controls, it is not a statistically significant difference (74.4 \pm 23.2, $P=.4$ ). On the CHQ-PF50, younger subjects scored significantly higher than healthy controls regarding behavior, mental health, social limits-physical, social limits-behavior/emotional, family activities, and the psychosocial summary score.

TABLE 5. CHQ-CF87 results $(n=8)$

\begin{tabular}{lccc}
\hline \multicolumn{1}{c}{ Health variable } & Score $( \pm$ SD) & $\begin{array}{c}\text { Normal } \\
\text { value }( \pm \text { SD) }\end{array}$ & $\boldsymbol{P}$ value \\
\hline General health perception & $76.4(18.3)$ & $66.4(14.6)$ & .08 \\
Physical functioning & $95.9(6.4)$ & $88.8(14.0)$ & .02 \\
Bodily pain & $68.8(26.4)$ & $74.4(23.1)$ & .5 \\
Behavior & $85.7(6.8)$ & $76.6(14.6)$ & $<.01$ \\
Mental health & $82.6(9.6)$ & $72.7(16.0)$ & .08 \\
Self-esteem & $84.8(23)$ & $81.8(14)$ & .6 \\
Social limits, physical & $100(0)$ & $88.3(21.0)$ & $<.001$ \\
Social limits, behavior & $97.2(7.8)$ & $86.5(21.5)$ & $<.01$ \\
Social limits, emotional & $93.1(13.2)$ & $85.9(21)$ & .33 \\
\hline Parameters without normative data were excluded from this table. SD, Standard de- \\
viation; $C H Q$ - $C F 87$, Child-Health Questionnaire Child-Completed 87 -Item Form.
\end{tabular}

Subjects older than 18 years also achieved a total score on the PedsQL significantly higher than healthy controls. On the SF-36v2, older subjects scored significantly higher than healthy controls regarding mental health, physical functioning, role physical, and vitality.

\section{DISCUSSION}

AAOCA is a rare congenital coronary variant with an increased risk of SCD, for which surgical repair is often recommended. ${ }^{1}$ We present the medium-term clinical results after AAOCA repair on the most young patients from a single institution. More important, more than half of our cohort experienced postoperative cardiac-type symptoms, and many of those symptoms were the same symptoms experienced preoperatively, including several subjects who continued to report exercise-induced symptoms. This raises the question of which symptoms should be used in consideration for referral for surgical repair. Furthermore, even if a patient presents with exercise-induced symptoms, if there is no evidence of

TABLE 6. CHQ-PF50 results $(n=8)$

\begin{tabular}{lccc}
\hline \multicolumn{1}{c}{ Health variable } & Score $( \pm$ SD) & $\begin{array}{c}\text { Normal } \\
\text { value }( \pm \text { SD) }\end{array}$ & $\boldsymbol{P}$ value \\
\hline General health perception & $72.3(15.9)$ & $73.0(17.3)$ & .91 \\
Physical functioning & $95.8(11.8)$ & $96.1(13.9)$ & .95 \\
Bodily pain & $88.8(16.4)$ & $81.7(19.0)$ & .3 \\
Behavior & $91.0(6.1)$ & $75.6(16.7)$ & $<.001$ \\
Mental health & $88.1(8.0)$ & $78.5(13.2)$ & .04 \\
Self-esteem & $87.4(12.8)$ & $79.8(17.5)$ & .22 \\
Social limits, physical & $100(0)$ & $93.6(18.6)$ & $<.0001$ \\
Social limits, behavioral or & $100(0)$ & $92.5(18.6)$ & $<.0001$ \\
$\quad$ emotional & $97.9(6.0)$ & $89.7(18.6)$ & $<.01$ \\
Family activities & $80.6(13.7)$ & $72.3(17.2)$ & .17 \\
Family cohesion & $95.8(11.8)$ & $87.8(19.9)$ & .26 \\
Time impact on parent & $82.2(12.1)$ & $80.3(19.1)$ & .77 \\
Emotional impact on parent & $54.6(2.2)$ & $53.0(8.8)$ & .1 \\
Physical Summary Score & $57.6(3.9)$ & $51.2(9.1)$ & $<.01$ \\
Psychosocial Summary Score & Shild-Health Questionnaire Parent-Completed \\
SD, Standard deviation; CHQ-PF50, Child & &
\end{tabular}


TABLE 7. SF-36v2 results $(n=4)$

\begin{tabular}{lccc}
\hline \multicolumn{1}{c}{ Health variable } & Score $( \pm$ SD) & $\begin{array}{c}\text { Normal } \\
\text { value }( \pm \text { SD) }\end{array}$ & $\boldsymbol{P}$ value \\
\hline General health & $53.2(5.8)$ & $50(10)$ & .52 \\
Mental health & $57.4(1.5)$ & $50(10)$ & $<.01$ \\
Physical functioning & $57.1(0.95)$ & $50(10)$ & $<.001$ \\
Role physical & $57.2(0)$ & $50(10)$ & $<.0001$ \\
Bodily pain & $58.8(3.7)$ & $50(10)$ & .08 \\
Vitality & $60.8(2.8)$ & $50(10)$ & .03 \\
Social functioning & $54.8(5.0)$ & $50(10)$ & .34 \\
Role emotional & $53.6(3.3)$ & $50(10)$ & .47 \\
Physical health summary & $57.0(3.2)$ & $50(10)$ & .16 \\
Mental health summary & $55.6(2.9)$ & $50(10)$ & .26 \\
\hline SD, Standrd
\end{tabular}

ischemia on cardiac testing, should these symptoms be used in consideration for surgical repair? Our study also documented a much higher incidence of postoperative symptoms than has been reported previously., ${ }^{8,20-22}$ This may be because we included both resting and exercise-induced symptoms. We chose to do this because several subjects presented with resting symptoms only, and these symptoms led to referral for cardiac evaluation and may have played a role in the decision-making process for repair. Interestingly, despite the frequency of persistent symptoms, subjects continue to enjoy an average or above average QOL. ${ }^{15}$

Our study supports that surgical correction for AAOCA can be done with extremely low mortality. However, two-thirds of our subjects experienced morbidity with the operation, with many developing pericardial effusions $(46 \%$ of this cohort) that required more frequent follow-up but rarely operative intervention. Although pericardial effusions occur commonly after open-heart surgery for congenital heart disease, larger series evaluating this issue have documented a lower prevalence than in our study. Cheung and colleagues ${ }^{23}$ documented with serial echocardiographic evaluations that the prevalence of postoperative pericardial effusion was $23 \%$ in a cohort of 336 patients undergoing open-heart surgery for congenital heart disease. Mild aortic insufficiency developed postoperatively in 4 subjects $(17 \%)$ with a trileaflet aortic valve. Of these, $3(75 \%)$ underwent aortic commissural resuspension during the operation, suggesting that this may be a surgical risk factor. Although no subject in our cohort has yet required an aortic valve intervention, which has been reported in other series, ${ }^{24}$ the long-term consequence of aortic insufficiency for these patients is unclear. Several of our findings are in contrast to other studies evaluating subjects with AAOCA postoperatively, ${ }^{8,13,21}$ perhaps because of differences in definitions of postoperative complications or morbidity.

In this same cohort of subjects previously evaluated for ischemia, ${ }^{14} 4$ additional subjects have had abnormal findings on ischemia testing, making a total of 12 subjects in our cohort who have had abnormal ischemia tests postoperatively. Two subjects with documented abnormalities in the short-term study had subsequent normal ischemia testing. Findings were inconsistent among the imaging modalities, and all subjects were asymptomatic during testing, questioning both the use of patient complaints in risk assessment for ischemia and the utility of follow-up with only one testing modality. The implication of these results on subsequent SCD risk is unclear and warrants further investigation.

Frequency of follow-up and type of testing performed at clinical visits were variable, and one-third of subjects were overdue for their routine cardiac evaluation by more than 1 year. It is unclear why such a large proportion of patients were not continuing with recommended clinical care but may be due to length of interval between visits (ie, 2 or 3 years), which may be too long for patients to remember, and the lack of adequate transition to adult care. This study highlights that more defined management guidelines for all patients with AAOCA are needed.

AAOCA subjects in this cohort scored in the normal range for all QOL parameters tested across the multiple tools used, frequently with scores significantly higher than healthy controls. Our results differ from other studies evaluating QOL in subjects with a history of congenital heart disease. Uzark and colleagues ${ }^{25}$ evaluated 347 children (aged 5-18 years) with congenital heart disease using the PedsQL child and parent-proxy report. They found that, by self-report, mean PedsQL scores for children with congenital heart disease were significantly lower than healthy norms for physical and psychosocial functioning across all age groups. Even among children with less severe cardiovascular disease, $19.2 \%$ reported significantly impaired psychosocial QOL. ${ }^{25}$ It is possible that patients with congenital heart disease diagnosed at birth or shortly thereafter may have been labeled as sick or fragile as a young child and, despite surgical correction, have had limitations imposed on them that have persisted and negatively affected their QOL. In contrast, subjects with AAOCA were otherwise healthy before diagnosis and the operation is thought of as curative. It is possible that these subjects perceive that they have returned to their healthy baseline, explaining the normal QOL scores.

Our study was limited by a small cohort size from a single institution but is the largest report of only pediatric patients with interarterial, intramural AAOCA in the medium-term after surgical repair. Because of the retrospective nature of the medical record review, we were limited to the most recent follow-up data for each subject. It is possible that we could be underestimating the frequency of symptoms and positive findings on ischemia testing, because many subjects were overdue for clinical evaluation. We sent QOL questionnaires to 21 subjects, with a return rate of 
$57 \%$. Although this is higher than average for survey response rates in organizational research, ${ }^{26}$ our sample size is small and it is unclear how a higher return rate would have affected our data analysis.

In summary, cardiac-type symptoms are frequent after surgical repair of AAOCA, with many subjects experiencing the same symptoms as before the operation, questioning the use of symptoms without evidence of ischemia as a determining factor for surgical referral. Although there was no surgical mortality, most of the cohort experienced morbidity with the operation, but despite this, subjects continue to experience average or higher than average QOL without exercise restriction. Future studies focusing on QOL in those who have not had surgical repair and who are exercise restricted may be of interest. Indeed, more children and young adults with AAOCA, both repaired and unrepaired, need to be followed up clinically over longer periods. We are hopeful that the multi-institutional AAOCA registry of the Congenital Heart Surgeons' Society ${ }^{27}$ can create more defined management guidelines for children and young adults with AAOCA and begin to answer the critical questions of whether this population can be risk stratified for myocardial ischemia and if SCD risk is diminished after surgical intervention.

\section{References}

1. Cheitlin MD, De Castro CM, McAllister HA. Sudden death as a complication of anomalous left coronary origin from the anterior sinus of Valsalva: a not so minor congenital anomaly. Circulation. 1974;50:780-7.

2. Yuksel S, Meric M, Soylu K, Gulel O, Zengin H, Demircan S, et al. The primary anomalies of coronary artery origin and course: a coronary angiographic analysis of 16,573 patients. Exp Clin Cardiol. 2013;18:121-3.

3. Angelini P, Velasco JA, Flamm S. Coronary anomalies: incidence, pathophysiology, and clinical relevance. Circulation. 2002;105:2449-54.

4. Chaitman BR, Lesperanc J, Salteil J, Bourassa MG. Clinical, angiographic, and hemodynamic findings in patients with anomalous origin of the coronary arteries. Circulation. 1976;53:122-31.

5. Lipsett J, Cohle SD, Berry PJ, Byard RW. Anomalous coronary arteries: a multicenter pediatric autopsy study. Pediatr Pathol. 1994;14:287-300.

6. Pelliccia A, Spataro A, Maron BJ. Prospective echocardiographic screening for coronary artery anomalies in 1,360 elite competitive athletes. Am J Cardiol. 1993;72:978-9.

7. Maron BJ, Doerer JJ, Haas TS, Tierney DM, Mueller FO. Sudden deaths in young competitive athletes: analysis of 1,866 deaths in the US, 1980-2006. Circulation. 2009;119:1085-92.

8. Mainwairing RD, Reddy VM, Reinhartz O, Petrossian E, MacDonald M, Nasiorov $\mathrm{T}$, et al. Anomalous aortic origin of a coronary artery: medium-term results after surgical repair in 50 patients. Ann Thorac Surg. 2011;92:691-7.

9. Frommelt PC, Frommelt MA, Tweddell JS, Jaquiss RD. Prospective echocardiographic diagnosis and surgical repair of anomalous aortic origin of a coronary artery from the opposite sinus with an interarterial course. J Am Coll Cardiol. 2003;42:148-54.

10. Davis JA, Cecchin F, Jones TK, Portman MA. Major coronary artery anomalies in a pediatric population: incidence and clinical importance. J Am Coll Cardiol. 2001;37:593-7.

11. Maron BJ, Shirani J, Poliac LC, Mathenge R, Roberts WC, Mueller FO. Sudden death in young competitive athletes. JAMA. 1996;276:199-204.

12. Eckart RE, Scoville SL, Campbell CL, Shry EA, Stajduhar KC, Potter RN, et al. Sudden death in young adults: a 25-year review of autopsies in military recruits. Ann Intern Med. 2004;141:829-34.

13. Turner II, Turek JW, Jaggers J, Herlong JR, Lawson DS, Lodge AJ. Anomalous aortic origin of a coronary artery: preoperative diagnosis and surgical planning. World J Pediatr Cong Heart Surg. 2011;2:340-5.

14. Brothers JA, McBride MG, Seliem MA, Marino BS, Tomlinson RS, Pampaloni $\mathrm{MH}$, et al. Evaluation of myocardial ischemia after surgical repair of anomalous aortic origin of a coronary artery in a series of pediatric patients. J Am Coll Cardiol. 2007;50:2078-82.

15. Brothers JA, McBride MG, Marino BS, Tomlinson RS, Seliem MA, Pampaloni MH, et al. Exercise performance and quality of life following surgical repair of anomalous aortic origin of a coronary artery in the pediatric population. J Thorac Cardiovasc Surg. 2009;137:380-4.

16. Varni JW, Burwinkle TM, Seid M. The PedsQL 4.0 as a school population health measure: feasibility, reliability, and validity. Qual Life Res. 2006;15:203-15.

17. Health ActCHQ. The CHQ Scoring and Interpretation Manual. Cambridge, MA: The Health Institute, New England Medical Center; 2008.

18. Ware JE Jr. SF-36 health survey update. Spine. 2000;25:3130-9.

19. Landraf JM, Abetz LN. Functional status and well-being of children representing three cultural groups: initial self-reports using the CHQ-CF87. Psychol Health. 1997; 12:839-54.

20. Davies JE, Burkhart HM, Dearani JA, Suri RM, Phillips SD, Warnes CA, et al. Surgical management of anomalous aortic origin of a coronary artery. Ann Thorac Surg. 2009;88:844-88.

21. Mumtaz M, Lorber R, Arruda J, Pettersson GB, Mavroudis C. Surgery for anomalous aortic origin of the coronary artery. Ann Thorac Surg. 2011;91:811-5.

22. Frommelt PC, Sheridan DC, Berger S, Frommelt MA, Tweddell JS. Ten-year experience with surgical unroofing of anomalous aortic origin of a coronary artery from the opposite sinus with an interarterial course. J Thorac Cardiovasc Surg. 2011;142:1046-51.

23. Cheung EWY, Ho SA, Tang KKY, Chau AKT, Chiu CSW, Cheung YF. Pericardial effusion after open heart surgery for congenital heart disease. Heart. 2003;89:780-3.

24. Romp RL, Herlong JR, Landolfo CK, Sanders SP, Miller CE, Ungerleider RM, et al. Outcome of unroofing procedure for repair of anomalous aortic origin of left or right coronary artery. Ann Thorac Surg. 2003;76:589-96.

25. Uzark K, Jones K, Slusher J, Limbers CA, Burwinkle TM, Varni JW. Quality of life in children with heart disease as perceived by children and parents. Pediatrics. 2008;121:e1060-7. doi: 10.154/peds.2006-3778.

26. Baruch Y, Holtom B. Survey response rate levels and trends in organizational research. Human Relations. 2008;61:1139-60.

27. Brothers JA, Gaynor JW, Jacobs JP, Caldarone C, Jegatheeswaran A, Jacobs ML. Anomalous Coronary Artery Working Group. The registry of anomalous aortic origin of the coronary artery of The Congenital Heart Surgeons' Society. Cardiol Young. 2010;25:50-8. 